\title{
Determination of Atomic Structure of the Metal-Oxide Interface: Pd Nanodeposits on an $\mathrm{FeO}(111)$ Film
}

\author{
Sh. K. Shaikhutdinov, ${ }^{*}$ R. Meyer, D. Lahav, M. Bäumer, T. Klüner, and H.-J. Freund \\ Department of Chemical Physics, Fritz-Haber-Institut der Max-Planck-Gesellschaft, Faradayweg 4-6, Berlin 14195, Germany
}

(Received 11 February 2003; published 13 August 2003)

\begin{abstract}
Palladium was vapor deposited on a thin $\mathrm{FeO}(111)$ film grown on a $\mathrm{Pt}(111)$ substrate. Scanning tunneling microscopy study has revealed that $\mathrm{Pd}$ wets the $\mathrm{FeO}$ substrate and at elevated temperatures forms extended $\operatorname{Pd}(111)$ monolayer islands in contrast to other oxide supports previously studied. For the first time, we have imaged the metal-oxide interface structure with atomic resolution and explained the results on the basis of ab initio calculations.
\end{abstract}

DOI: 10.1103/PhysRevLett.91.076102

PACS numbers: 68.35.Bs, 68.35.Md, 68.37.Ef, 68.47.Jn

The metal-oxide interface is important in a variety of areas in nanoscience. Metal-oxide-metal structures play a decisive role in microelectronics; nanoparticles on oxide supports are key structures in catalysis; in photonics, the collective properties of metal clusters deposited on oxide substrates depend on the dielectric response of the interface $[1,2]$.

Various techniques such as high-resolution electron microscopy, x-ray photoelectron diffraction (XPD) and $\mathrm{x}$-ray absorption spectroscopies have been utilized in order to study the metal-oxide interface structure on an atomic level $[3,4]$. Recently, scanning tunneling microscopy (STM) has atomically imaged metal particles deposited on oxide substrates. Hansen et al. have shown that Pd particles on a thin alumina film mainly expose the (111) facets [5]. Dulub et al. [6], studying the $\mathrm{Pt} / \mathrm{TiO}_{2}(110)$ system, have resolved the surface of large Pt particles which have been encapsulated by a reduced titanium oxide layer. However, for determining the metaloxide interface structure it is necessary to resolve both the metal and oxide structure simultaneously.

In addition to experimental works, theoretical modeling of oxide supported metal clusters has recently started. For example, preferential adsorption sites of transition metals on oxide supports such as $\mathrm{Pd}$ on $\mathrm{MgO}(100)$, $\mathrm{TiO}_{2}(110), \alpha-\mathrm{Al}_{2} \mathrm{O}_{3}(0001)$ surfaces have been calculated ([7-12], and references therein). The calculations also explained a three-dimensional growth mode for Pd on various oxide surfaces as observed experimentally [13-20]. However, Goniakowski and Noguera [21] have recently predicted that $\mathrm{Pd}$ must wet the $\mathrm{MgO}(111)$ surface [in contrast to the $\mathrm{MgO}(100)$ surface] due to strong interaction with the polar (111) surface.

In the present Letter, we have investigated Pd deposited onto a thin $\mathrm{FeO}(111)$ film grown on a $\mathrm{Pt}(111)$ substrate. For the first time, we have observed that $\mathrm{Pd}$ wets the $\mathrm{FeO}$ surface in accordance with theoretical predictions for metals deposited on (111) rock-salt surfaces and report atomically resolved STM images of the metal-oxide interface. STM data supported by theoretical calculations show that Pd atoms adsorb on on-top sites on the oxygen layer.

The structure of the FeO film formed on $\mathrm{Pt}(111)$ has been previously studied by Galloway et al. [22,23], Fadley et al. [24,25], and Weiss et al. [26,27]. It has been shown that the oxide film consists of closely packed oxygen and iron layers and is terminated by oxygen [24]. The distance between $\mathrm{O}$ and $\mathrm{Fe}$ layers measured by XPD is $\sim 0.68 \AA$ [25], which is much smaller than for the bulk $\mathrm{FeO}(\sim$ $1.25 \AA$ ). The oxygen anions occupy threefold hollow sites on the Fe layer. The $\sim 10 \%$ lattice mismatch between $\mathrm{FeO}$ layer and underlying $\operatorname{Pt}(111)$ gives rise to a moiré pattern with a periodicity ca. $26 \AA$.

For the $\mathrm{FeO}$ film preparation, one monolayer (ML) of $\mathrm{Fe}$ was evaporated onto a clean $\mathrm{Pt}(111)$ substrate at $300 \mathrm{~K}$ and then oxidized in ca. $10^{-6} \mathrm{mbar}^{\mathrm{O}_{2}}$ at $1000 \mathrm{~K}$ for $2 \mathrm{~min}$. The quality of the $\mathrm{FeO}$ film was checked by observation of a characteristic low energy electron diffraction (LEED) pattern [22,26,27] and by STM. (All STM images discussed in this Letter were taken at room temperature.) The STM images showed wide terraces separated by steps of $\sim 2.3 \AA$ in height, corresponding to monoatomic steps of $\operatorname{Pt}(111)$ underneath the film. The terraces exhibited a long-range surface modulation (a moiré pattern) as well as an atomic structure with a $\sim 3 \AA$ lattice constant. Small holes in the film but no point defects (such as vacancies) were observed [28].

Palladium deposition at $130 \mathrm{~K}$ results in a random nucleation of the Pd particles, as shown in Fig. 1(a). No preferential decoration of the steps edges or holes is observed. The lateral size of the Pd deposits increases with increasing coverage and reaches ca. $5 \mathrm{~nm}$ at $0.5 \mathrm{ML}$ [28]. However, the particles always show the height $\sim 2.3 \AA$, that is equal to the interlayer distance in the bulk $\operatorname{Pd}(111)(=2.26 \AA)$. The structure of the Pd deposits is resolved in the inset in Fig. 1(a), which shows the (111) symmetry of the Pd deposits. Therefore, we can conclude that $\operatorname{Pd}(111)$ islands of one atomic layer thick are formed on the $\mathrm{FeO}$ film at submonolayer coverages. 

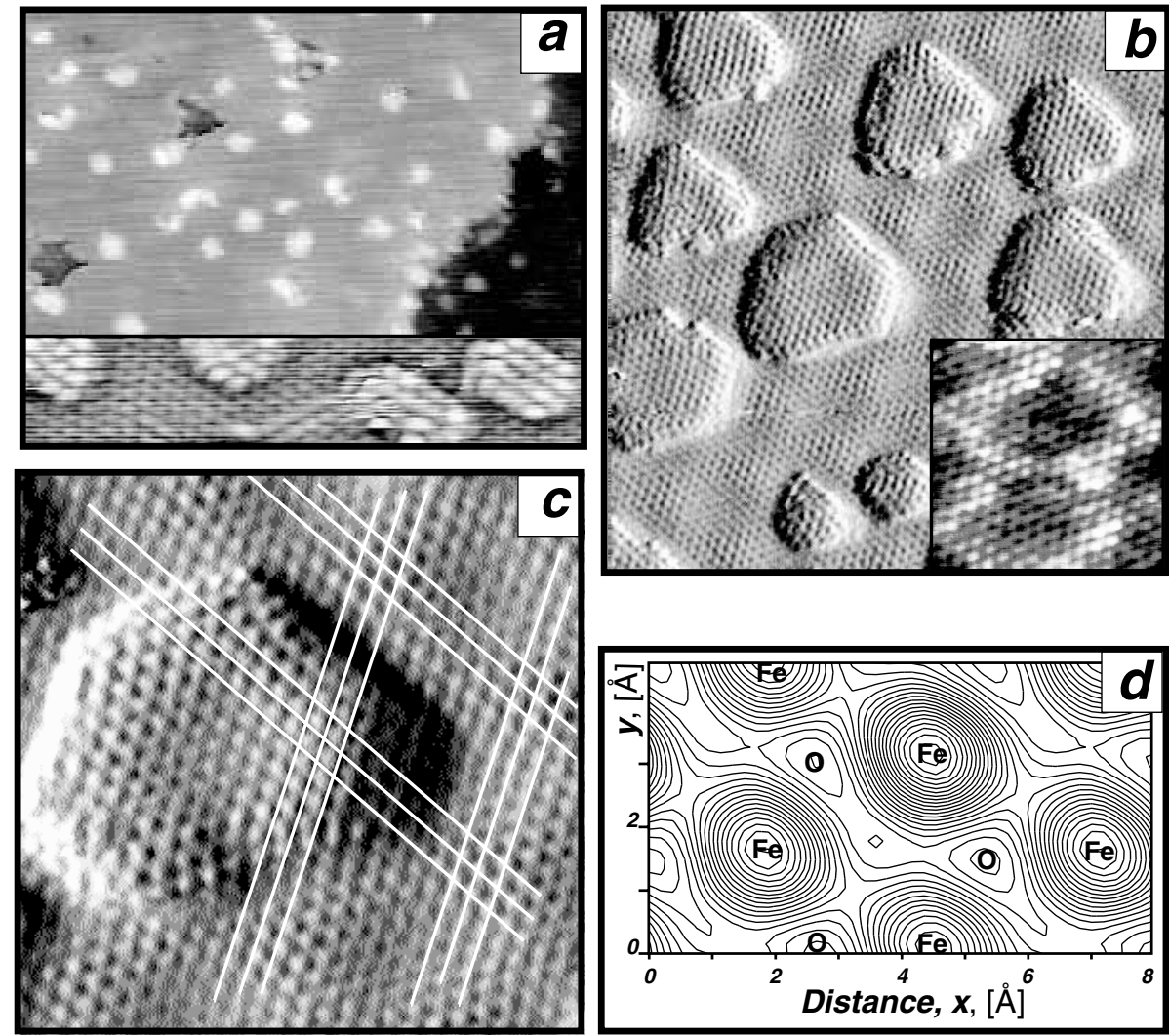

FIG. 1. (a) STM image of $0.05 \mathrm{ML}$ Pd deposited on a FeO film at $130 \mathrm{~K}$ shows a random nucleation of the particles (size $39 \times$ $22 \mathrm{~nm}^{2}, V_{\text {tip }}=200 \mathrm{mV}, I=2 \mathrm{nA}$ ). The atomic structure of the Pd deposits exhibiting the (111) surface is resolved in the inset $(-9 \mathrm{mV}, 1.8 \mathrm{nA})$. (b) STM image, presented in differentiated contrast, of the extended Pd(111) islands formed by deposition of $0.5 \mathrm{ML}$ of $\mathrm{Pd}$ at $130 \mathrm{~K}$ and subsequent annealing to $600 \mathrm{~K}\left(20 \times 20 \mathrm{~nm}^{2}, 2 \mathrm{mV}, 4.5 \mathrm{nA}\right)$. The surface of the large Pd(111) islands exhibits a long-range modulation as shown in the inset $\left(4.8 \times 4.8 \mathrm{~nm}^{2},-20 \mathrm{mV}, 5 \mathrm{nA}\right)$. (c) Registry analysis of the $\mathrm{Pd}(111) / \mathrm{FeO}(111)$ interface shows that $\mathrm{Pd}$ atoms occupy hollow sites over the lattice of protrusions imaged on $\mathrm{FeO}\left(6 \times 6 \mathrm{~nm}^{2}\right.$, $2 \mathrm{mV}, I=5 \mathrm{nA}$ ). (d) Contour plot of the lowest unoccupied DOS near Fermi level for the FeO film showing the maxima over the Fe ions.

Campbell and co-workers [29] have recently suggested that formation of two-dimensional (2D) metal islands on oxides may occur below a certain coverage due to kinetic limitations. As the coverage and/or temperature increases, the metal islands irreversibly thicken, thus forming thermodynamically more favorable threedimensional (3D) particles. However, we have observed that, at increasing coverage, the next layer starts to grow after the first layer gets nearly completed [28]. Moreover, annealing to $600 \mathrm{~K}$ results in Pd spreading on the oxide surface such that extended Pd islands are formed at 0.5 ML [see Fig. 1(b)]. Therefore, our results clearly indicate wetting of $\mathrm{Pd}$ on $\mathrm{FeO}$.

One might argue that the oxide layer may encapsulate metal deposits at elevated temperatures due to the lower surface energy of the oxide as compared to Pd. However, thermal desorption spectra of $\mathrm{CO}$ as a probe molecule are found to be similar to those obtained for Pd crystalline surfaces. Therefore, we assign the observed deposits to Pd islands.

High-resolution STM images, displayed in Figs. 1(b) and 1(c), show that the $\mathrm{Pd}(111)$ islands exhibit triangular and hexagonal shapes typical for the epitaxial growth on the close-packed (111) surfaces. The fact that both metal and substrate are atomically resolved allows us to perform a registry analysis. The analysis shows that the protrusions on the Pd surface occupy hollow sites with respect to the lattice of protrusions imaged on the $\mathrm{FeO}$ surface as illustrated in Fig. 1(c). Note that this result is observed to be independent of the bias polarity.

Interestingly, the Pd surface exhibits a moiré-like modulation, as shown in the inset in Fig. 1(b). This could be explained by surface buckling [30] of the underlying $\mathrm{FeO}$ layer, which is retained on the Pd surface since the atoms within the $\operatorname{Pd}(111)$ islands are in registry with the $\mathrm{FeO}$ substrate.

In order to interpret STM images and understand the nature of the $\mathrm{Pd} / \mathrm{FeO}(111) / \mathrm{Pt}(111)$ interface, we have performed density functional theory (DFT) calculations. In our calculations, we applied a commercial version of the CASTEP [31] program available as a module of CERIUS ${ }^{2}$ [32]. The $\mathrm{FeO} / \mathrm{Pt}(111)$ film was simulated by a slab model containing four layers of $\mathrm{Pt}$ and one layer of $\mathrm{Fe}$ and $\mathrm{O}$ stacking as O-Fe-Pt(4). In order to reduce the numerical 
effort of the calculations, the lattice constant of the $\operatorname{Pt}(111)$ substrate was extended from 2.77 to $3.09 \AA$ in order to match the $\mathrm{FeO}(111)$ layer. Galloway et al. [23] applied a similar approach in their calculations and found this approximation to be acceptable. Therefore, a slab model with three atoms per layer could be constructed.

The PBE exchange-correlation functional [33] was used and the core electrons were replaced by ultrasoft nonlocal pseudopotentials [34]. A plane wave cutoff of $E_{\text {cut }}=400 \mathrm{eV}$ and a surface Brillouin zone sampling containing eight special $k$-points were used. Also, no structural relaxation of the Pt substrate has been considered.

As a first step, we have performed a full geometry optimization of $\mathrm{FeO}$ layer. The calculations show that Fe ions occupy fcc threefold hollow sites on $\operatorname{Pt}(111)$, and oxygen prefers threefold hollow sites on the Fe layer. The Pt-Fe interlayer distance is predicted to be $1.89 \AA$. An Fe-O interlayer distance of $0.63 \AA$ has been calculated which corresponds to a $\mathrm{Fe}-\mathrm{O}$ interatomic distance of $1.86 \AA$. The results are in good agreement with previous calculations of Galloway et al. $(0.65 \AA / 1.90 \AA)$ [23] and XPD experiments $(0.68 \AA / 1.91 \AA)$ [25].

Based on the analysis of the lowest unoccupied states near the Fermi level calculated for the optimized geometry, we have simulated the STM image of the FeO film as shown in Fig. 1(d). The contour plot clearly shows that the highest density is observed over the Fe atoms as the main contribution comes from the 3D orbitals of iron. Therefore, the protrusions in the STM images of the

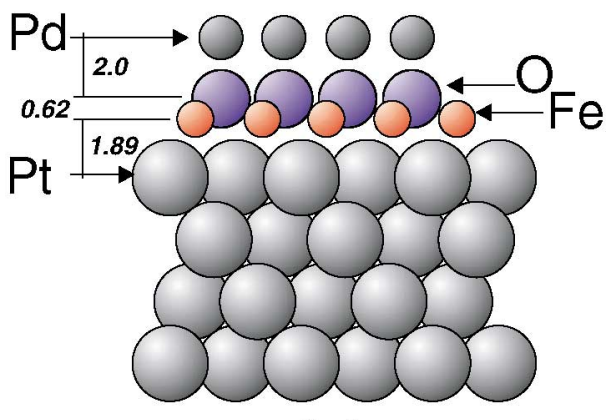

(a)

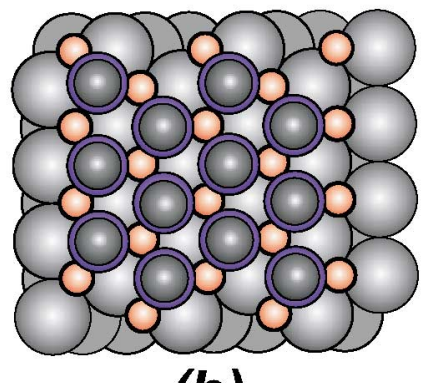

(b)

FIG. 2 (color online). Side (a) and top (b) views of the $\mathrm{Pd} / \mathrm{FeO} / \mathrm{Pt}$ structure as determined from DFT calculations. The interlayer distances (in $\AA$ ) are indicated.
FeO film must be assigned to the position of the Fe atoms. As the protrusions within $\mathrm{Pd}(111)$ islands occupy the hollow sites over protrusions on the $\mathrm{FeO}$ surface, these calculations suggest that Pd atoms sit in the hollow sites with respect to the Fe ions. However, as seen in Fig. 1(d), there are two different sites possible: (i) on top of oxygen and (ii) a fcc hollow site on the O layer. Since the STM images cannot discriminate them, we have performed calculations where a Pd monolayer has been added on top of the O-Fe-Pt(4) slab.

The calculations show that the presence of the $\mathrm{Pd}$ monolayer does not affect the support geometry as depicted in Fig. 2. Indeed, the $\mathrm{Fe}-\mathrm{O}$ interlayer distance $(0.62 \AA)$ is nearly equal to that calculated for the pristine $\mathrm{FeO} / \mathrm{Pt}$ system $(0.63 \AA)$. The Pd-O interlayer distance is predicted to be $\sim 2.00 \AA$, which agrees fairly well with the STM results showing the height of Pd islands of $\sim 2.3 \AA$.

The most stable geometry has been calculated where Pd occupies the on-top sites on the oxygen layer, with the adsorption energy being $2.7 \mathrm{eV} /$ atom. If the Pd adsorption is restricted to take place on the fcc hollow sites of the $\mathrm{O}$ layer and on top of iron, then the structure exhibits a total energy ca. 0.7 and $0.3 \mathrm{eV} /$ atom higher, respectively, as compared to the adsorption on top of oxygen. Therefore, the calculations agree well with STM results, assuming that Pd adsorbs on top of oxygen on the $\mathrm{FeO}(111)$ film.

Previous theoretical works have also shown that $\mathrm{Pd}$ prefers adsorption on top of oxygen on $\mathrm{MgO}(100)[7,8]$, on the bridge oxygen of the $\mathrm{TiO}_{2}(110)$ surface [9], over oxygen on the Al-terminated $\alpha-\mathrm{Al}_{2} \mathrm{O}_{3}(0001)$ surface [10], and on top of oxygen on the O-terminated thin alumina films formed on $\mathrm{Al}(111)$ and $\mathrm{NiAl}(110)$ [11]. Based on the DFT calculations of the $\mathrm{Pd} / \mathrm{MgO}(100)$ and $\mathrm{Pd} / \mathrm{Al}_{2} \mathrm{O}_{3}(0001)$ systems, respectively, Goniakowski [7] and Bogicevic et al. [11] explained the preferential adsorption of Pd on top of oxygen by an increased lateral polarization of the metal overlayer as opposed to a local interaction of $\mathrm{Pd}$ atoms at the fcc hollow site. For the $\mathrm{Pd} / \mathrm{FeO}(111) / \mathrm{Pt}(111)$ system studied here, we have found a similar effect as demonstrated in Figs. 3(a) and 3(b) by charge density difference plots.

Plot (a) shows that charge density between Pd atoms increases. In addition, the $\mathrm{Pd}-d_{z 2}$ and $\mathrm{O}-p_{z}$ orbitals are depleted. This reduces the Pauli repulsion between the metal layer and support and hence favors a stronger attractive interaction. Meanwhile, Pd adsorption over iron results in a positive charge difference between $\mathrm{Pd}$ and $\mathrm{Fe}$, thus leading to a weaker interaction.

The difference between $\mathrm{Pd}$ adsorption on top of $\mathrm{O}$ and $\mathrm{Fe}$ is also seen by analyzing the projected density of the $\operatorname{Pd} d$ states as shown in Fig. 3(c). The center of gravity of the $d$-band shifts to lower energies with respect to the Fermi level for adsorption on top of oxygen as compared to adsorption over iron. This leads to a better stabilization of Pd $d$ states and a lower total energy. 

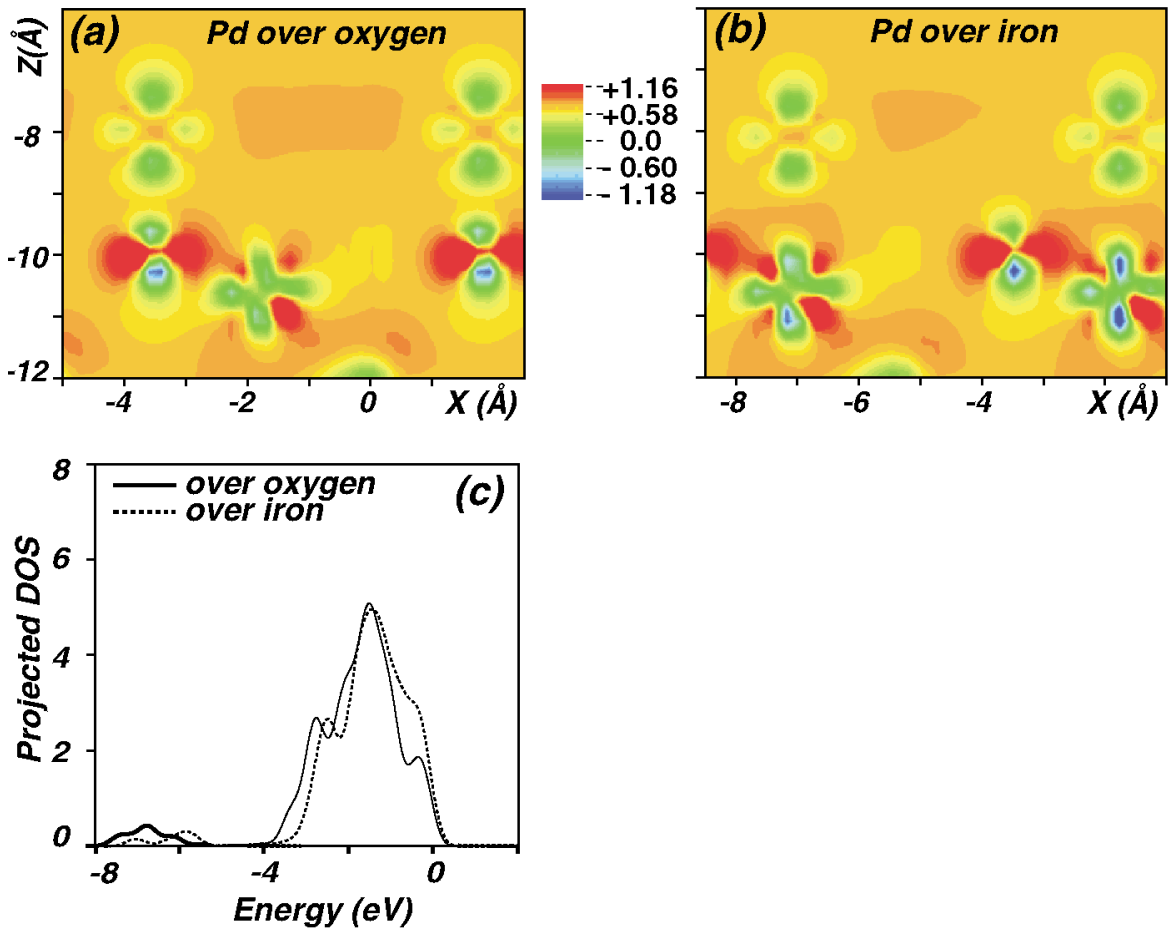

FIG. 3 (color). The cross view of the charge density difference distribution for Pd on top of oxygen (a) and over iron (b). (c) Projected DOS for Pd sitting on top of oxygen (solid) and over iron (dotted).

In summary, we have shown that Pd deposited on an $\mathrm{FeO}(111)$ thin film grown on $\mathrm{Pt}(111)$ forms extended $\operatorname{Pd}(111)$ islands of one atomic layer thick. This is in line with the predicted wetting behavior of the rock-salt (111) surfaces. We have atomically resolved the metal-oxide interface structure via STM and performed ab initio calculations of the $\mathrm{Pd} / \mathrm{FeO}$ system corroborating the experimental results.

The authors thank Georg Kresse for fruitful discussions. This work was supported by Fonds der Chemischen Industrie. One of us (R.M.) thanks Alexander von Humboldt Foundation for support.

*Corresponding author: shaikhutdinov@fhi-berlin.mpg.de

[1] C. R. Henry, Surf. Sci. Rep. 31, 231 (1998).

[2] H.-J. Freund, Surf. Sci., 500, 271 (2002).

[3] R. Schweinfest et al., Philos. Mag., 81, 927 (2001).

[4] K. van Betthem et al., Mikrochim. Acta 138, 181 (2002).

[5] K. H. Hansen et al., Phys. Rev. Lett. 83, 4120 (1999).

[6] O. Dulub, W. Hebenstreit, and U. Diebold, Phys. Rev. Lett. 84, 3646 (2000).

[7] J. Goniakowski, Phys. Rev. B 57, 1935 (1998).

[8] L. Giordano, J. Goniakowski, and G. Pacchioni, Phys. Rev. B 64, 75417 (2001).

[9] T. Bredow and G. Pacchioni, Surf. Sci. 426, 106 (1999).

[10] J. R. B. Gomes et al., J. Chem. Phys. 116, 1684 (2002).

[11] A. Bogicevic and D. Jennison, Phys. Rev. Lett. 82, 4050 (1999).

[12] Z. Lodziana and J. K. Norskov, Surf. Sci. Lett. 518, L577 (2002).
[13] M. Bäumer and H.-J. Freund, Prog. Surf. Sci. 61, 127 (1999).

[14] M. Heemeier et al., Surf. Sci. 523, 103 (2003).

[15] J. B. Giorgi et al., Surf. Sci. Lett. 498, L71 (2002).

[16] C. L. Pang, H. Paza, S. A. Hayock, and G. Thornton, Surf. Sci. 460, L510 (2000).

[17] C. Xu et al., J. Vac. Sci. Technol. A 15, 1755 (1997).

[18] C. Xu, X. Lai, G.W. Zajac, and D.W. Goodman, Phys. Rev. B 56, 13464 (1997).

[19] M. J. J. Jak et al., Surf. Sci. 474, 28 (2001).

[20] P. Stone et al., Surf. Sci. 433, 501 (1999).

[21] J. Goniakowski and C. Noguera, Phys. Rev. B 66, 085417 (2002).

[22] H. C. Galloway, J. J. Benitez, and M. Salmeron, J. Vac. Sci. Technol. A 12, 2302 (1994).

[23] H. C. Galloway, P. Sautet, and M. Salmeron, Phys. Rev. B 54, R11 145 (1996).

[24] C. S. Fadley et al., J. Electron Spectrosc. Relat. Phenom. 75, 273 (1995).

[25] Y. J. Kim et al., Phys. Rev. B 55, R13 448 (1997).

[26] M. Ritter, W. Ranke, and W. Weiss, Phys. Rev. B 57, 7240 (1998).

[27] W. Ranke, M. Ritter, and W. Weiss, Phys. Rev. B 60, 1527 (1999).

[28] R. Meyer, M. Bäumer, Sh.K. Shaikhutdinov, and H.-J. Freund (to be published).

[29] C. T. Campbell, Surf. Sci. Rep. 27, 1 (1997).

[30] Sh. K. Shaikhutdinov, M. Ritter, and W. Weiss, Phys. Rev. B 62, 7535 (2000).

[31] M. C. Payne et al., Rev. Mod. Phys. 64, 1045 (1992).

[32] CERIUS ${ }^{2}$ is provided by Accelrys., Inc.

[33] J. P. Perdew, K. Burke, and M. Ernzerhof, Phys. Rev. Lett. 77, 3865 (1994).

[34] D. Vanderbilt, Phys. Rev. B 41, 7892 (1990). 